\title{
A Robust Route Maintenance Scheme for Wireless Ad-Hoc Networks
}

\author{
Kwan-Woong Kim ${ }^{1}$, Mike Myung-Ok Lee ${ }^{2}$, ChangKug Kim ${ }^{3}$, and Yong-Kab Kim ${ }^{1}$ \\ ${ }^{1}$ Div. of Electrical Electronic \& Information Eng., \\ Wonkwang Univ., Iksan, 570-749, South Korea \\ \{watchbear, ykim\} @wonkwang.ac.kr \\ ${ }^{2}$ Murdoch University, South Street, Murdoch, Western Australia 6150, Australia \\ Mike. Lee@murdoch. edu. au \\ ${ }^{3}$ Bioinformatics Div, National Institute of Agricultural Biotechnology, R.D.A. \\ 225 Seodundong, Suwon, 441-707, Korea
}

\begin{abstract}
Ad hoc networks are dynamic networks that consist of mobile nodes. Nodes in Ad hoc networks are usually laptops, PDAs or mobile phones. These devices feature Bluetooth and/or IEEE 802.11 (WiFi) network interfaces and communicate in a decentralized manner. Due to characteristics of Ad-hoc networks, Mobility is a key feature of routing protocol design. In this study, we present an enhanced routing maintenance scheme that cope with topology changes pre-actively. The key feature of the proposed scheme is that switches next-hop node to alternative neighbor node before link breakage for preventing route failure. From extensive experiments by using NS2, the performance of the proposed scheme has been improved by comparison to AODV protocol.
\end{abstract}

Keywords: Wireless Ad-hoc Networks, Routing Protocols, Mobility, AODV.

\section{Introduction}

Wireless Ad-hoc network [1,2] is a self-organized, dynamically changing multi-hop network. All mobile nodes in an ad-hoc network are capable of communicating with each other without the aid of any established infrastructure or centralized controller. Ad-hoc network is useful in many applications because they do not need any infrastructure support and has capability of self configuration. Sensor networks, disaster recovery, rescue and automated battlefields are examples of application environments.

The nodes have the responsibility of self-organizing so that the network is robust to the variations in network topology due to node mobility as well as the fluctuations of the signal quality in the wireless environment.

Compared to traditional routing protocols in wired networks, that of Ad Hoc networks required to cope with the high rate of topology changes.

This implies that the routing protocol should propagate topology changes and compute updated routes to the destination. Since wireless ad-hoc networks usually have limited bandwidth and battery power, their routing protocols should have low control overhead. Reactive or on-demand routing protocols have been developed for this reason. In an on-demand routing protocol, a node only maintains routes for in-use 
destinations and does not pro-actively advertise routes. Rather, it queries for needed routes and offers routes in response to queries. Dynamic Source Routing (DSR), Adhoc On-demand Distance Vector Routing (AODV)[3], Lightweight Mobile Routing (LMR), Temporally Ordered Routing Algorithm (TORA), Associativity-Based Routing (ABR)[4], Signal Stability Routing (SSR) [5] are classified to on-demand scheme.

Table-driven protocols attempt to continuously update routes within the network so that when a packet needs to be forwarded, the route is already known and can immediately be used. The family of distance-vector or link-state algorithms is examples of table-driven schemes. There is Destination-Sequence Distance Vector (DSDV), Wireless Routing Protocol (WRP), Clusterhead Gateway Switch Routing (CGSR).[5]

Nodes in MANET may move freely and unpredictably, the path that packets traverse to its destination will be broken by link failure frequently. Link breakage caused by node mobility may degrade overall performance.

Tom Goff proposed preemptive-routing protocol that is an enhanced version of AODV and DSR [6]. Preemptive routing protocol measures receiving signal power to make decision whether launch re-route discovery before link breakage occurred by node mobility. Path recovery of this is similar with hand-off in cellular networks. Some on-demand protocols with multi-paths or backup routes have been proposed to improve the performance in ad-hoc networks. AODV-BR scheme improves AODV routing protocols by constructing a mesh structure and providing multiple alternate routes. The algorithm establishes the mesh and multi-path using the RREP of AODV, which does not transmit many control messages.[7][8]

In this work, we proposed a novel route maintenance scheme based on AODV that takes node mobility into consideration.

This paper structured as follows. In section 2, we present about related works and background. The proposed routing protocol based on AODV is presented in section 3 . In section 3, Simulation results obtained by the proposed scheme are evaluated. Finally, section 4 presents conclusions and discussion.

\section{Proposed Route Maintenance Scheme}

Route maintenance in routing protocols plays a role of maintaining route connectivity and link breakage detection. In AODV, local route repair algorithm can be used for fast route recovery [3][9]. But most of existing routing algorithms have lack of ability route recover before link breakage occurred.

Our work is focus on routing maintenance to prevent route-failure which caused by node mobility and improve efficiency of routing protocol during forwarding packets. Our approach is quite different with other mobility support routing protocols.

To achieve this goal, Routing protocol should have ability of local route change from moving node to alternative node. The basic idea of the proposed scheme is based on our previous work [10].

The distance between two nodes is inverse proportional to the receiving signal strength $R x P$ at the receiver in wireless networks [11]. If $R x P$ is being lower, transmitting node moves far, else if $R x P$ is increasing, then it moves near. 
In previous studies, the method presented that estimates relative speeds of two nodes by measuring $R x P$ variation. Prior to estimate relative speeds, the distance between two nodes must be known. But it is not easy to extract distance from $R x P$. Since $R x P$ is composed of several factors such as transmitting power, antenna gain and channel loss factor [11], node might not have enough information.

In the proposed scheme, we use receiving signal variation function $V$ for detecting node movements as follows.

$$
V=R x P\left(t_{1}\right)-R x P\left(t_{0}\right)
$$

If function $V$ is negative value, it indicates two adjacent nodes becoming far, else if $V$ is equal to zero, two nodes doesn't move or move same direction with the same speed. When value $V$ is positive, two adjacent nodes move closer.

\section{Process of the Proposed Scheme}

In the proposed scheme, transmission range is divided into two zones, GREEN ZONE and RED ZONE as shown in figure 2. The next hop node locates in GREEN ZONE; it supposes to be safe state for data communication.

If node located in RED ZONE, the proposed scheme can be triggered for route reconstruction.

Figure 1 illustrates the first phase of the proposed scheme. When intermediate node (' $C$ ') receives data packet from previous hop node 'A', it monitors receiving signal power $R x P$ and signal strength variation $V$.

If $R x P$ is under RxTh and $V(A)$ is over than 0 (movement detected), then node triggers local route change process to find alternative node among its neighbors. Looking for alternative nodes, node broadcasts HELP message to its one-hop neighbors. Where $R x T h$ is receiving power threshold for RED ZONE and defined as follows.

$$
R x T h=K \times R x P_{\min }
$$

$R x P_{\min }$ is the minimum receivable power by network interface device (e.g. $3.65 \times 10^{-10}$ Watts in $\left.802.11 \mathrm{~b}[12]\right)$ and $K$ is constant and set to 5 .

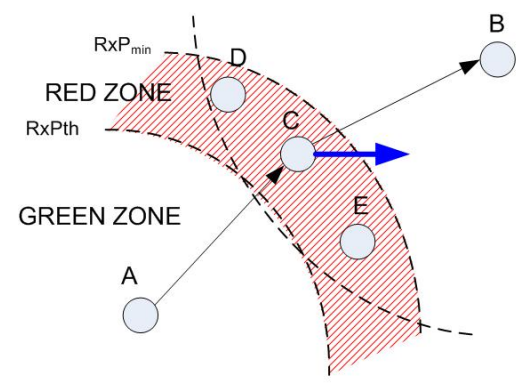

(a) node $C$ is moving and detect signal power falling during forwarding packets from $A$ to $B$

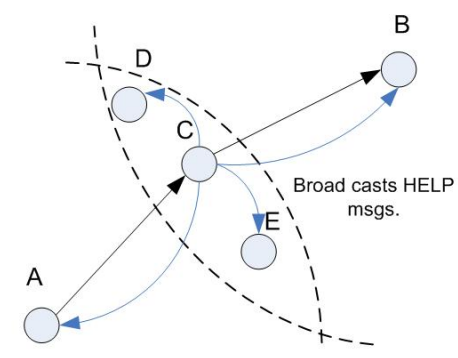

(b) node $\mathrm{C}$ broadcasts HELP messages to it's one-hop neighbor for Local Route Change

Fig. 1. The example of the proposed scheme: Broadcasts HELP message to its one-hop neighbor 


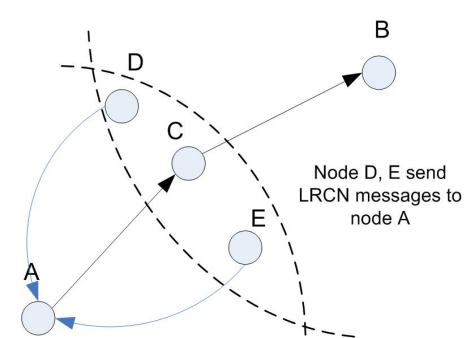

(C) Node A waiting for LRCN messages; node $D, E$ send $L R C N$ messages to node $A$

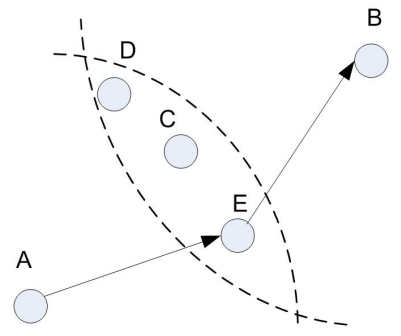

(D) Node A select node $E$ to next hop and forwards next packet to node $\mathrm{E}$

Fig. 2. The example of the proposed scheme: Previous-hop node receives LRCN message and switch next-hop node 'C' to new one

\begin{tabular}{|c|c|c|}
\hline Type & Reserved & Hop Count \\
\hline Prev-hop IP Address (32bits) \\
\hline Next-hop IP Address (32bits) \\
\hline \multicolumn{2}{|c|}{ Destination IP Address (32bits) } \\
\hline Destination Sequence Number (32bits) \\
\hline V (32bits) \\
\hline
\end{tabular}

(a) HELP message

\begin{tabular}{|l|c|c|}
\hline Type & Reserved & Hop Count \\
\hline Alternative node IP Address (32bits) \\
\hline \multicolumn{2}{|c|}{ Destination IP Address (32bits) } \\
\hline \multicolumn{2}{|c|}{ V (32bits) } \\
\hline
\end{tabular}

(b) LRCN message

Fig. 3. New message formats for the proposed scheme

Subsequently, a node receives HELP packets, under condition that previous node ' $\mathrm{A}$ ' and next hop node ' $\mathrm{B}$ ' are belong to its neighbor. If signal variation $V(A)$ is zero, node itself can be alternative of node $C$. Otherwise HELP message is ignored. Alternative node updates route information with HELP messages and sends LRCN (Local Route Change Notification) to previous node of HELP message. The previous hop node 'A' may receive HELP from its next hop node ' $C$ '. The node 'A' set timer for waiting LRCN (Local Route Change Notification) messages. When LRCN message are received, previous-hop node cancel timer and updates next-hop address ' $\mathrm{C}$ ' to source address of LRCN ' $E$ '. When the timer expired, the node initiates local route repair [3] process to re-establish path to destination.

New message formats are shown in figure 3 . V field is 32 bit floating point value.

Pseudo code of the proposed scheme procedures is shown bellows. To avoid unnecessary broadcasting HELP, node set the flag of precursor of route entry to 1 . It indicates HELP sent already for the flow. Precursor list is a set of nodes that share the same route to reach final destination [3]. The flag of precursor initialized when route is updated.

\section{Procedure of receiving DATA packet from node $\mathbf{i}$}

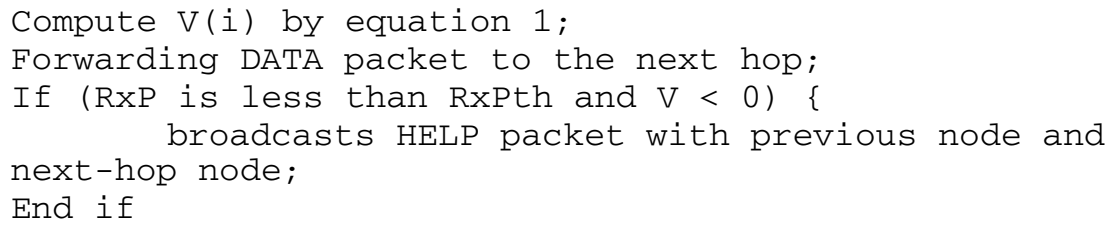



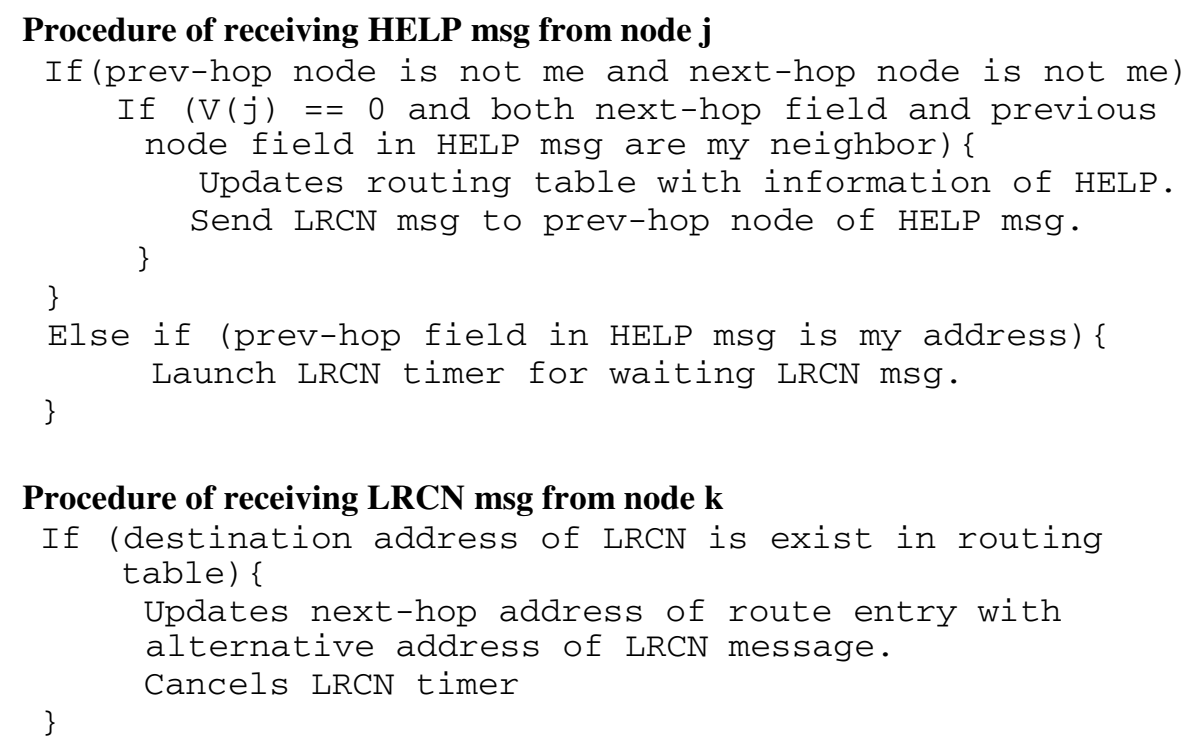

Procedure of LRCN timer expired

Launch local route repair

\section{Performance Evaluation}

In this section, a performance of the proposed routing protocol is evaluated using extensive simulations and compared its performance with AODV. NS2 simulator was used for experiments and the proposed scheme is implemented as part of AODV in NS2 [13]. The network model used for simulations consists of 100 mobile nodes in $1.0 \mathrm{~km} \times 1.0 \mathrm{~km}$ area. The initial position of nodes is randomly chosen. Node pairs are randomly selected to generate CBR/UDP traffic. Channel bandwidth is $2 \mathrm{Mbps}$.

Each node uses IEEE 802.11 MAC protocol and the used Channel model is Wireless channel/Wireless Physical propagation model. Two-Ray Ground model is used for radio propagation model and transmission range and interference range of a mobile node is $250 \mathrm{~m}$ and $550 \mathrm{~m}$. Traffic source are CBR (Constant Bit Rate) and 15 CBR sources generate UDP packet in every $0.1 \mathrm{sec}$. The size of UDP packet is 512 bytes. The simulation time is set to 200 seconds. The mean pause time of nodes is $10 \mathrm{sec}-$ onds. Maximum speed of nodes varies from $5 \mathrm{~m} / \mathrm{sec}$ to $20 \mathrm{~m} / \mathrm{sec}$.

To avoid the bias of random number generation, we performed simulation 10 times under the same configuration. Table 1 shows parameter of energy model in NS2.

Table 1. Parameter of Energy Model in NS2

\begin{tabular}{l|l|l} 
Attribute & Description & Value \\
\hline -initialEnergy & Given energy for each node & 200 Joules \\
\hline -Grx, Gtx & Antenna Gain & $\mathbf{1}$ \\
\hline -txPower & Transmitting power in Watt & $\mathbf{2 8 1 . 8 m W}$
\end{tabular}


Figure 4 plots end-to-end packet delivery ratio and number of lost packets respectively. As the maximum speed of nodes increase, more packets are dropped in the network by broken paths. In case of AODV, packet delivery ratio falls down significantly in high mobility situation. But AODV with proposed scheme keeps certain levels above $90 \%$.

In all cases, the proposed scheme improves the number of received packets and reduces packet loss. The main reason of performance improvement is that the proposed scheme can change route to alternative node before the next-hop node move out of transmission range. It could reduce packet loss and route failure more efficiently in high mobility environments.

From these results, AODV combined with the proposed scheme can give quite positive effects for overall performance and efficiency of route discovery.

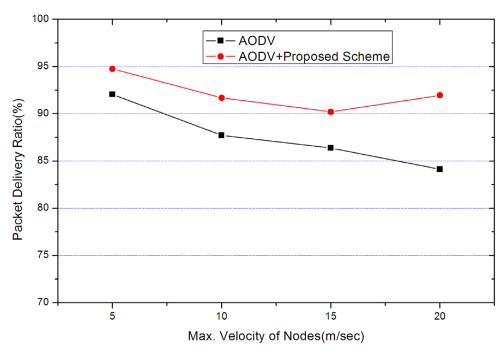

(a)

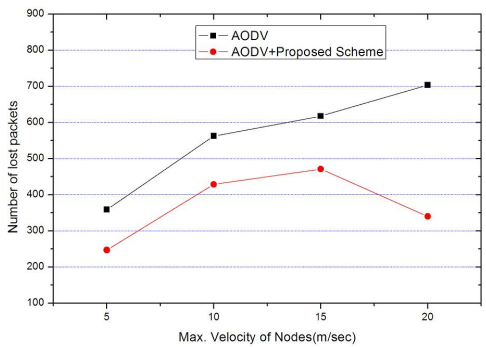

(b)

Fig. 4. (a) Packet delivery ratio. (b) Number of lost packets.

Performance comparison in control overhead and number of route discovery is shown in figure 5. In the most cases, AODV with proposed scheme reduces control message overhead and number of route discovery compared to AODV. It is obvious that local route change after route establishments can reduce probability of re-route discovery and control overhead efficiently.

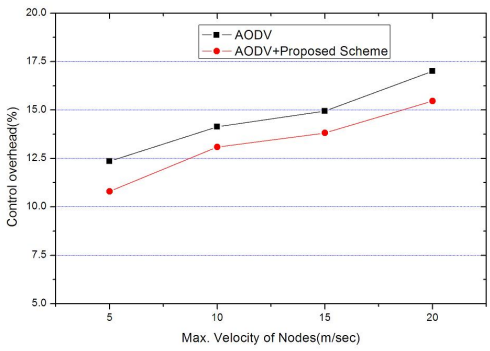

(a)

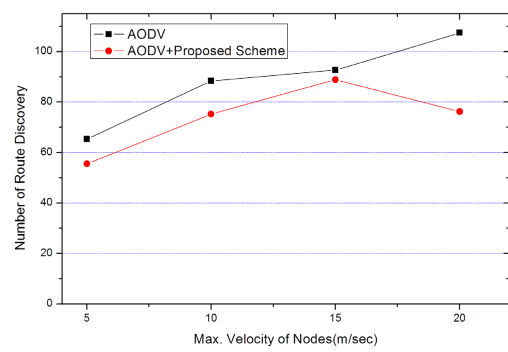

(b)

Fig. 5. (a) Comparison of control overhead. (b) Number of route discovery. 


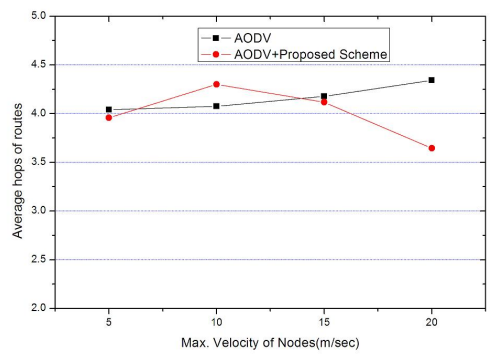

(a)

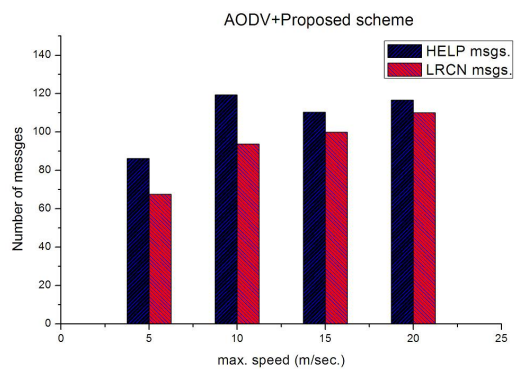

(b)

Fig. 6. (a) Average hops of routes. (b) Number of new control messages.

Figure 6 (a) depicts average hops of routes and number of transmitted new messages shown in figure 6(b). In the proposed scheme, previous hop node launches local route repair process when it failed to receive LRCN messages. Therefore hop counts of route can be increase in some cases. As maximum speed of node increase, the more HELP and LRCN message generated as shown in figure 6 (b).

In general, the results are quite positive in the sense that the proposed scheme outperformed than AODV in terms of routing overhead and throughput. Using our technique, the proposed scheme may reduce re-route discovery as well as overall end-toend throughput improvements over multi-hop ad-hoc networks.

\section{Conclusion}

Since the cost of detecting and re-establish broken path is high. The method for overcome mobility of node is one of main research issues in routing protocol design. In this paper, we presented a new route maintenance scheme for AODV using receiving signal variation. The main feature of the proposed scheme is capability of switching the next hop node to one of its available neighbour before the next hop node move out of transmission range. Additional messages are defined and the proposed scheme is implemented in network simulator NS2 for performance evaluation. From simulation results, the proposed scheme can reduce broken path, overhead of control messages, and improve end-to-end packet delivery ratio, which compared to AODV.

Acknowledgement. This paper was supported by Wonkwang University in 2006.

\section{References}

1. Perkins, C.E.: Ad Hoc Networking. Addison-Wesley, Upper Saddle River, NJ, USA, 1 (2001).

2. http://www.ietf.org/, IETF MANET Working Group.

3. Perkins, C.E., Royer, E.M., Das, S.R.: Ad-hoc on demand distance vector routing. IETF RFC3561, http://www.ietf.org/rfc/rfc 3561.txt, (2003) 
4. Toh, C.K.: Associativity Based Routing for Ad Hoc Mobile Networks. Wireless Pers. Commun. J. Special Issue on Mobile Networking and Computing Systems, vol. 4, no. 2, 3 (1997)

5. Elizageth, M., Royer, Toh, C.K.: A Review of Current Routing Protocols for Ad Hoc Mobile Wireless Networks, IEEE personal Communications, 4 (1999)

6. Goff, T., Nael, B., et al.: Preemptive Routing in Ad-hoc Networks. ACM SICMOBILE. 6(2001) 43-52

7. Lee, S.J., Gerla, M.: AODV-BR: Backup routing in Ad Hoc networks. Proceedings of IEEE WCNC 2000. Chicago, IL, (2000).

8. Wang, Y.H., Chuang, C.C., Hsu, C.P., Chung, C.M.: Ad hoc on demand routing protocol setup with backup routes. Proceedings of ITRE 2003. International Conference on Information Technology Research and Education, 8 (2003) 137-141.

9. Kim, K.H., SEO, H.G.: The Effects of Local Repair Schemes in AODV-Based Ad Hoc Networks. IEICE TRANSACTIONS on Communications, Vol.E87-B No.9, 8(2004)24582466,

10. Brahma, M.K., Kim, W., Abouaissa A., Lorenz, P.: A Load-Balancing and Push-Out Scheme for Supporting QoS in MANETs. Telecommunication Systems Journal, Vol. 30, No.1-3, 10(2005)161-175

11. Anderson, J.B., Rappaport, T.S., Yoshida, S.: Propagation Measurements and Models for Wireless Communications Channels. IEEE Communication Magazine, 1(1995) 42-49

12. WaveLAN/PCMCIA Card User's Guide - Lucent Technologies

13. Network Simulator: NS2.29 available via website http://www.isi.edu/nsnam.ns/ 